\title{
Three Dimensional Computation of Force in a Novel Brushless DC Linear Motor
}

\author{
A. Basak and A. F. Flores Filho \\ Wolfson Centre for Magnetics Technology, Cardiff School of Engineering \\ P.O. Box 917, Cardiff CF2 1XH, Wales UK \\ T. Nakata and N. Takahashi \\ Dept. Elec. and Elect. Eng., Okayama University \\ Okayama 700, Japan
}

\begin{abstract}
The flux distribution and static force of a novel brushless dc linear motor were calculated by $3 \mathrm{~d}$ finite element analysis. An analytical model to increase the force to volume ratio of the motor was validated by the numerical analysis as well. Experimental and numerical results present an excellent closeness.
\end{abstract}

\section{INTRODUCTION}

A novel brushless dc linear motor, Fig. 1, was developed. On its slider there are five NdFeB-type magnets which provide excitation flux. The travel length of the motor is 200 $\mathrm{mm}$. Its ferromagnetic circuit is made of annealed mild steel. The armature windings comprehend a set of independent coil sections positioned along the long armature bars [1][2][3]. The equipotential lines of the resultant magnetic field follow three orthogonal directions throughout the volume of the motor. So to analyse the flux distribution and the static force a $3 \mathrm{~d}$ finite element analysis required.

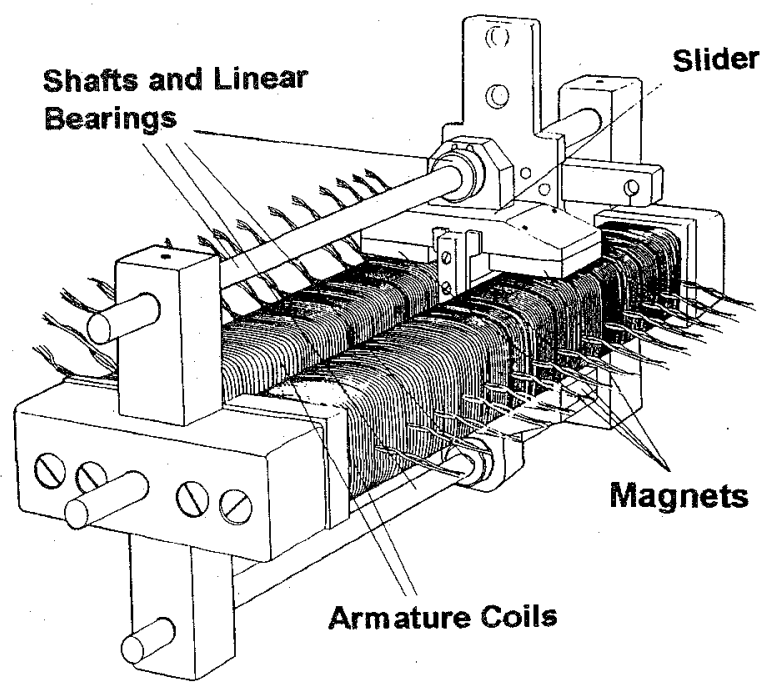

Fig. 1 - Perspective view of the linear motor

II. ANALYSIS

The ideal equation of the electromagnetic force on the slider can be obtained from the Lorentz's law as follows:

$$
\vec{F}=3 \int_{0}^{\vec{l}_{c}}\left(\frac{N w_{m}}{l_{t}}\right) \vec{B}_{g} \times I d \vec{l}_{c}
$$

In Eq.(1), $\vec{F}$ is the electromagnetic force on the slider, $N$ the total number of turns per coil section, $w_{m}$ is the width of the magnets, $l_{t}$ is the length of the coil sections, $I$ is the armature current, $\vec{l}_{c}$ is the active conductor length vector for each side of the coil and in the same direction of $I$, and $\vec{B}_{g}$ is the magnetic flux density in the airgap. The relation between the permanent magnet flux density and its airgap flux density is

$$
B_{g}=\frac{B_{m}}{L_{f} C_{f}}=\frac{B_{m}}{L_{f}}\left(\frac{S_{m}}{S_{g}}\right)
$$

In Eq. (2), $B_{m}$ is the permanent magnet flux density, $L_{f}$ and $C_{f}$ are respectively the flux leakage and flux fringing coefficients, $S_{m}$ is the cross section of the permanent magnet, while $S_{g}$ is the cross section of the airgap. $\vec{B}_{g}$ is considered to be orthogonal to $\vec{l}_{C}$. Hence Eq. (1) can be re-written in a scalar form:

$F=3\left(\frac{N w_{m}}{l_{t}}\right) I l_{\mathcal{C}} \frac{B_{m}}{L_{f} C_{f}}$

The expressions for the slider velocity $v$ and for the electromagnetic power $P_{e}$ are:

$v=\left(V_{t}-I r_{a}\right) /\left[3\left(\frac{N w_{m}}{l_{t}}\right) B_{g} l_{c}\right]$

and

$P_{e}=3\left(\frac{N w_{m}}{l_{t}}\right) B_{g} l_{c} v l$

In Eq.(4), $V_{t}$ is the voltage supplied to the armature coil, and $r_{a}$ is the resistance of the latter.

\section{FINTTE ELEMENT ANALYSIS}

The $3 \mathrm{~d}$ model of the linear motor was created by extruding a base plane along the direction of the travel length. For the analysis of flux distribution produced by the magnets only, it was possible to model the prototype by using only $1 / 2$ of its whole volume. The volume of the entire motor was discretised, together with a surrounding volume of air, in 73,744 brick-type elements creating 75,285 nodes. A different finite element model was created for each chosen position of the slider along the travel length. The actual $B H$ 


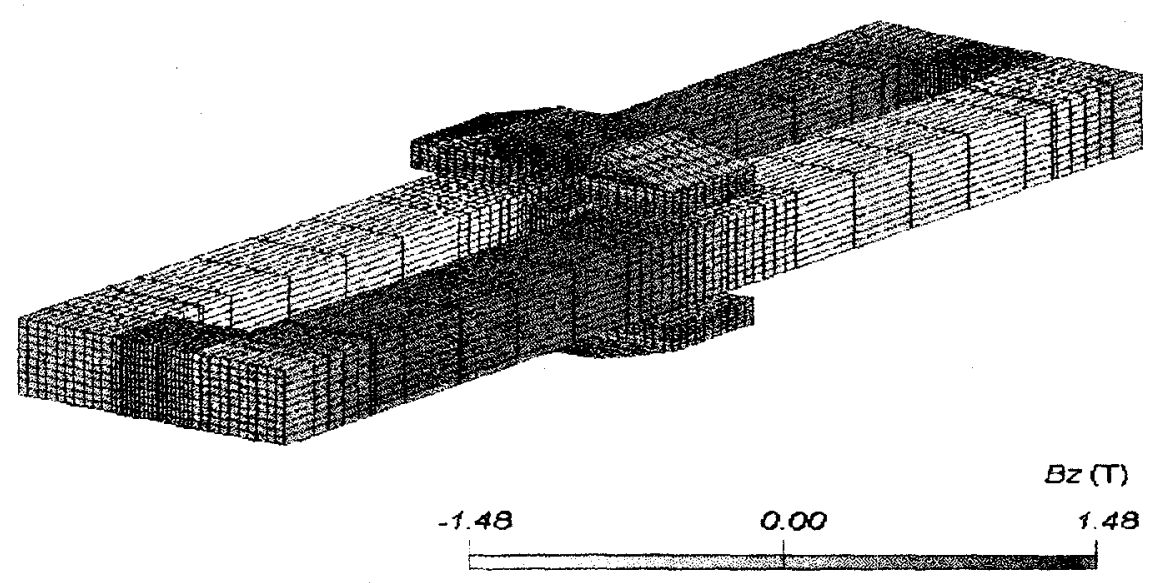

Fig. 2(a). $B_{\tau}$-oomponent plotting for the model without coils

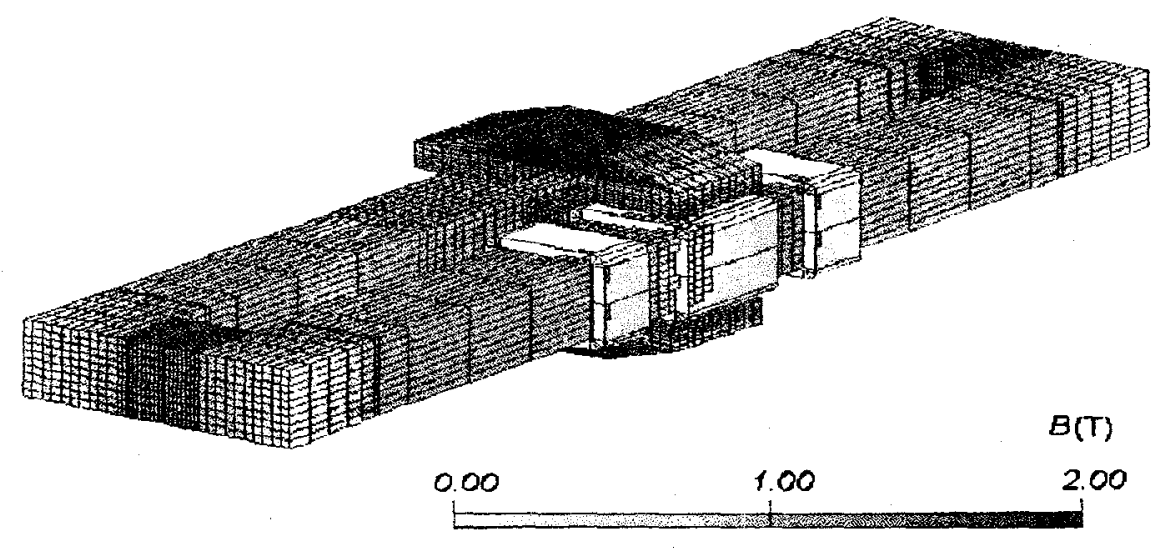

Fig. 2(b). Plotting of $B$ on the model with compensating coils and $I=4.5 \mathrm{~A}$

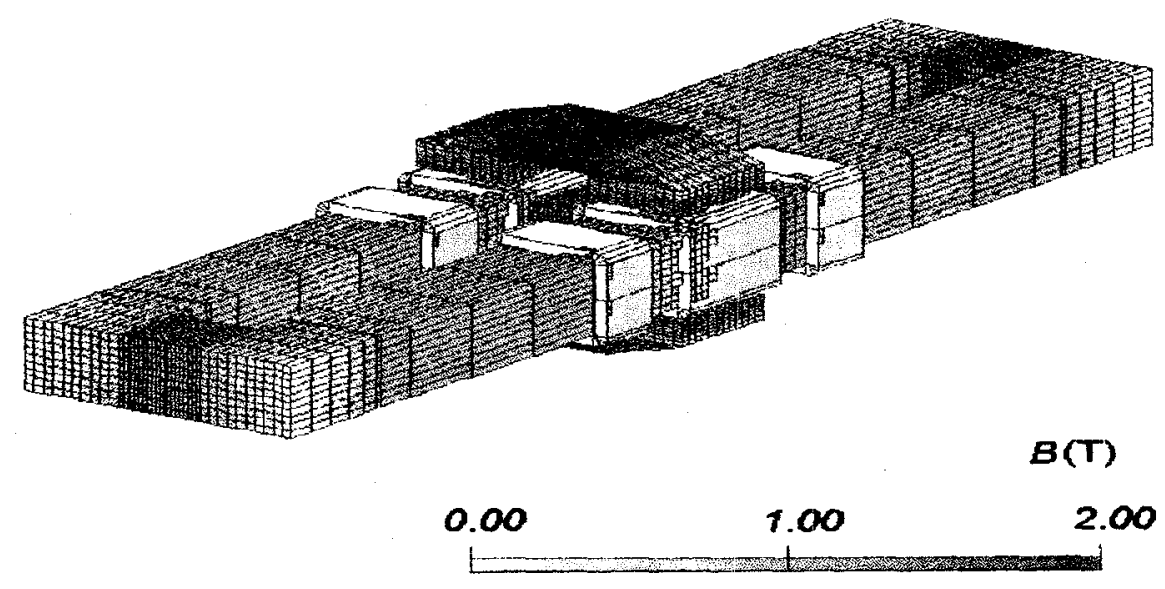

Fig. 2(c). $B$ plotting on the model with compensating coils, double armature adtion and $I=4.5 \mathrm{~A}$ 


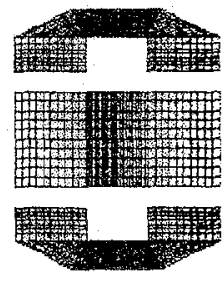

$B x y=\left(6 x^{* * 2} 2+B y^{* 2}\right) * 0.5(T)$

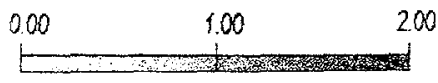

Fig. 2(d). Plotting of $B_{x y}$ on the slider

curves of the permanent magnets and the mild steel were employed to define the magnetic materials which the linear motor is made from. Figures 2(a) to (d) show the flux density plotting on the volume of the different models analysed. The air volumes were set to have a reduced scalar potential.As the armature magnetic circuit was closed and had no airgaps, the setting of a small volume of it as reduced scalar potential was necessary The force developed by the motor was also simulated; the Maxwell's stress tensor was employed.

\section{COMPUTATIONAL RESILTS}

Shape functions were used to calculate the iron and airgap flux density. In presence of current, the integral coil technique was also employed. The computation of the flux distribution and force was performed in seven models, each one for a different position of the slider.

\section{A. Comparison with Experimental Results}

To measure the airgap flux density in several airgaps presented in the motor, a Hall effect digital Gaussmeter was used. The measurements were taken at specific points, i.e. at the centre of the faces of the magnets by the airgaps. A good correlation was found between the numerical values of airgap flux density and the correspondent measured ones. The maximum difference was found to be $9 \%$. The measurement of the airgap flux densities was taken in model with slider at half way, i.e. at $z=0.00$. In Table 1 , the airgap flux density for the model with annealed mild steel and with no current is presented.

TABLE 1-Comparative table of the theoretical, measured and simnlated (FEM) airgaps flux densities for model with $I=0$.

\begin{tabular}{|l|c|c|c|}
\hline \multirow{2}{*}{ Probe position } & \multicolumn{3}{|c|}{ Flux density (mT) } \\
\cline { 2 - 4 } & Theoretical & Measured & FEM \\
\hline face of magnet 1 & 461 & 506 & 529 \\
\hline face of magnet 2 & 461 & 516 & 529 \\
\hline face of magnet 3 & 461 & 508 & 529 \\
\hline face of magnet 4 & 461 & 508 & 529 \\
\hline left face magnet 5 & 461 & 542 & 543 \\
\hline right face magnet 5 & 461 & 563 & 563 \\
\hline
\end{tabular}

The assessment of the flux density data in Table 1 indicates an excellent correlation between the experimental data and numerical ones. The largest difference is, in this case, $4.5 \%$. The static force the motor develops was measured along the travel length by means of a load cell attached to the slider. The position zero corresponds to halfway travel length. Figure 2 show the values of computed and measured force.

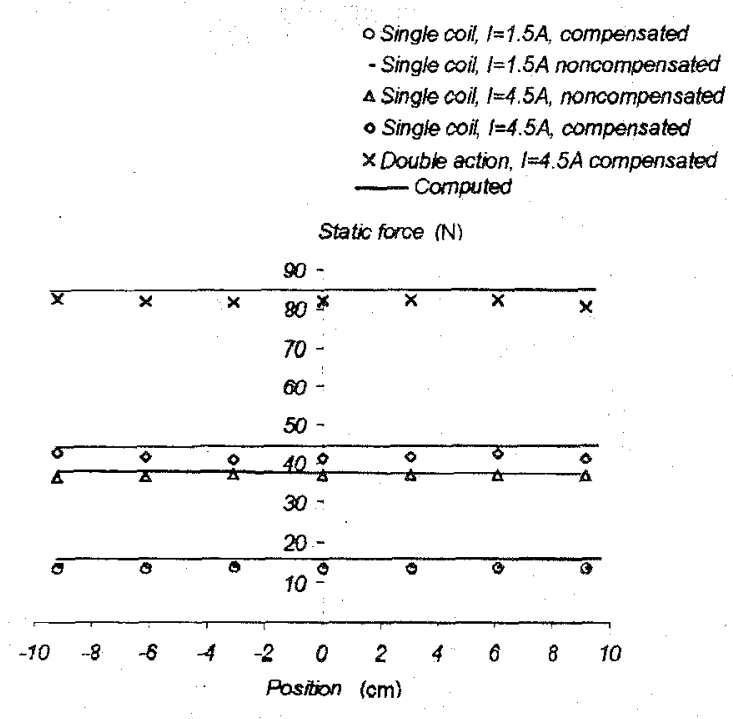

Fig. 2. Static force along the travel length

\section{Conclusions}

The use of $3 \mathrm{~d}$ finite element computation to simulate the flux distribution and the static force produced by the novel brushless dc linear motor has proved not only necessary but efficient and accurate. The linear motor presents a measured sensitivity that goes up to 18.2N/A for the model operating with double action and $I=4.5 \mathrm{~A}$. The use of the coil sections of the linear motor as compensating coils is not only a practical solution to avoid saturation, but it also proved effective to increase the force the motor can produce.

\section{ACKNOWLEDGEMENT}

Mr. Flores Filho wishes to thank the Brazilian agency, namely CAPES, and the Dep. of Electrical Engineering of the Federal University of Rio Grande do Sul, where he is a lecturer, for the support to his work.

\section{REFERENCES}

[1] Basak and A. F. Flores Filho, "Design, analysis and test of a novel slotless linear stepping motor," ICEM, Paris, France, Proceedings vol. 2, pp. 98100, September 1994.

[2] Basak and A. F. Flores Filho, "Design Optimisation of a Novel Slotless Linear Stepping Motor," ICEMD, Durham, UK, September 1995.

[3] Basak and A. F. Flores Filho, "Investigation of a Novel Double Armature Brushless D.C. Linear Motor," 30th Annual Mecting of the IEEE IAS, Oriando, Flo, USA, October 1995. 